\title{
Estimation of Flow Behavior on Rock Joints Using the Depth Averaged Flow Model
}

\author{
"Prosper MGAYA, ${ }^{* *}$ Kiyoshi KISHIDA, ${ }^{* * *}$ Takashi HOSODA, and ${ }^{* * *}$ Akitaka YAMAMOTO \\ "PhD Student, Department of Urban Management, Kyoto University (606-8501, Kyoto) \\ ${ }^{* *}$ Associate Professor, Department of Urban Management, Kyoto University (606-8501, Kyoto) \\ ${ }^{* * *}$ Professor, Department of Urban Management, Kyoto University (606-8501, Kyoto) \\ ${ }^{* * *}$ Kajima Corporation (Tokyo-Japan)
}

\begin{abstract}
A study has been carried out on estimation of flow in rock joint using depth-averaged flow model. Based on the results from an idealized sinusoidal rock joint roughness, it is found that the hydraulic conductivity of rock joints is strongly affected by both aperture and joint surface roughness. The model proposed herein has shown its ability in simulating the flows on rock joints especially for small shear displacement with comparison to experimental results. However, it is recommended that more research has to be carried to investigate on how the effect of wall roughness deformation due to shear displacement can be accommodated in the model.
\end{abstract}

Key Words: rock joint, depth averaged flow model, joint roughness

\section{Introduction}

Flow past rock joint has attracted considerable research attention because of the central role they play in several intriguing phenomena such as stability and performance of underground structures especially nuclear waste disposal issue. Therefore, it is important to grasp the hydro-mechanical behavior of rocks underneath. This includes understanding the behavior of permeability (hydraulic conductivity) of the single rock joint under different geometry properties.

Several studies on flows in rock joint investigating the effects of geometry characteristics using an idealized sinusoidal and or Sawtooth patterns have been conducted ${ }^{1}$. These studies made possible to have some analytical solutions expressing the relationship between hydraulic conductivity, fracture geometry variation and stresses acting on rocks basing on empirical relations ${ }^{1,2}$. However they could not give details on how, the flow variables such as pressure and shear resistance respond to geometry variations. On the other hand, by applying the shear mechanical model of rocky joint, the shear behavior and the variation of joint surface roughness on rock joint have been clarified ${ }^{3,4}$.

However in all effort made, there exists few mathematical models that can simulate the flow behavior in rock joints and understand the response of flow resistance from theoretical point of view. As result more research depends on experimental results.

The present study is concerned with simulation of flow in rock joint using the depth-averaged flow model. An idealized sinusoidal wall roughness of the rock joint is firstly assumed for model calibration ${ }^{1}$. Also the behavior of flow variables such discharge, shear resistance and pressure distribution is studied for different roughness of an idealized sinusoidal pattern. Thereafter the model is applied to physical rock joint specimen and then comparison is made between the model and experimental results.

The numerical model developed herein is based on Reynolds- depth averaged Navier-Stokes equations which is verified by analytical solution of one-dimensional flow model.

\section{Laboratory tests}

The simultaneous direct shear and permeability tests were carried in consideration of joint surface roughness and material properties under constant normal confining conditions $^{5,6}$. The outline of the experimental apparatus is shown in Fig.1. 


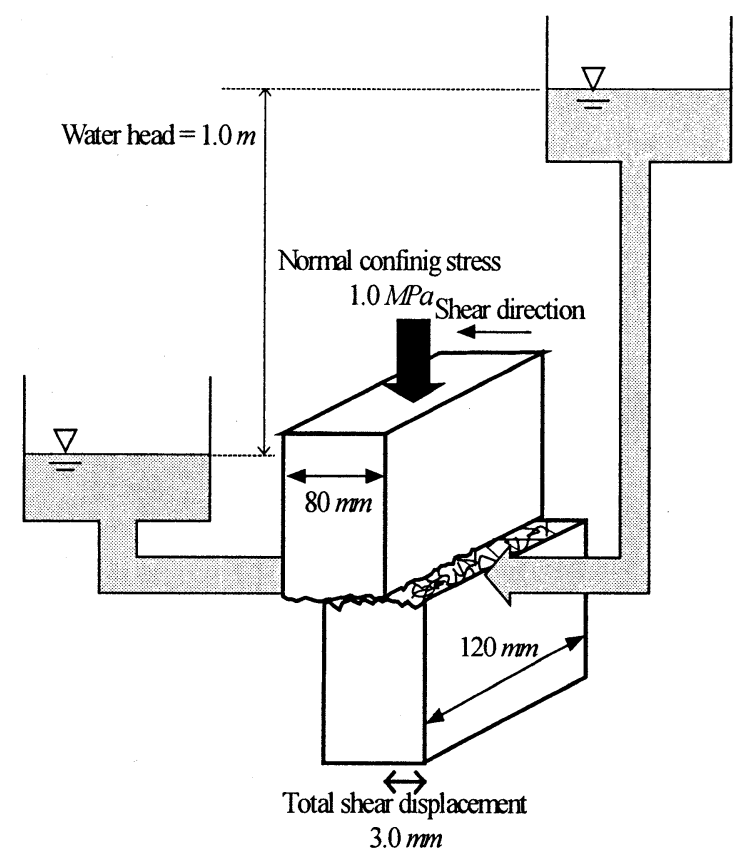

Fig.1 Schematic setup of permeability test experiment

\subsection{Outline of the laboratory tests}

The experiment was carried out according to the standard method used in several studies for permeability test, where shear displacement, dilation, shear stress, normal stress, transmissibility, and pore pressure, can be measured $^{5,6}$.

In this study, the hydraulic-head and the normal confining stress were kept constant at $1.0 \mathrm{~m}$ and 1.0 $\mathrm{MPa}$ respectively. The permeability tests were performed at each predetermined shear displacement up to $3 \mathrm{~mm}$.

Before performing the tests, measurements of the joint surface roughness were taken with a roughness profiler at an interval of $0.25 \mathrm{~mm}$.

\subsection{Typical results}

Figure 2 and 3 shows the effect of joint surface roughness and aperture size with regard to transmissibility of the joint. It is observed that transmissibility increase with advance of shear displacement as well as aperture size.

Also it is important to note that there exists an increase of transmissibility with increase of $J R C$ (joint surface roughness) at the same value of shear displacement. However this is noticed at higher values of shear displacement in this case beyond $0.5 \mathrm{~mm}$.

$J R C$ is number that can be estimated by comparing the appearance of a discontinuity surface with standard profiles ${ }^{7} . J R C=0$ corresponds to totally smooth surface and $\mathrm{JRC}=20$ corresponds to a very rough surface

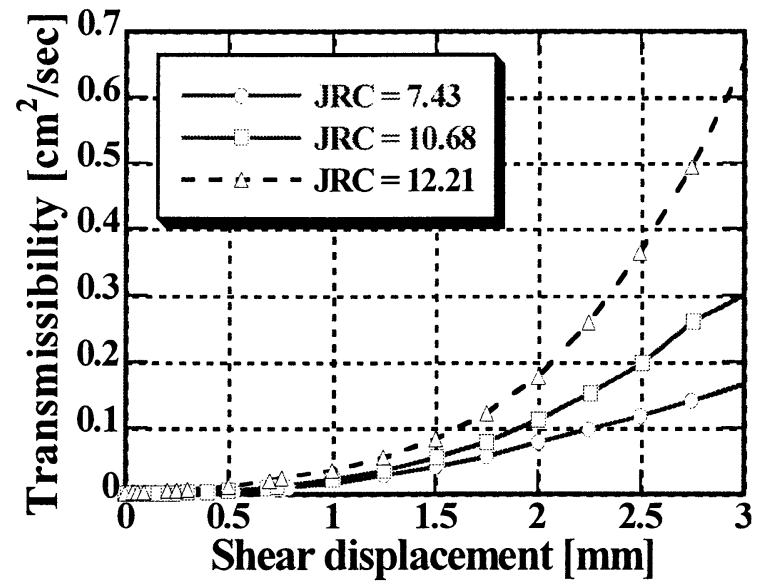

Fig.2 The variation of joint transmissibility with respect to both shear displacement and joint roughness

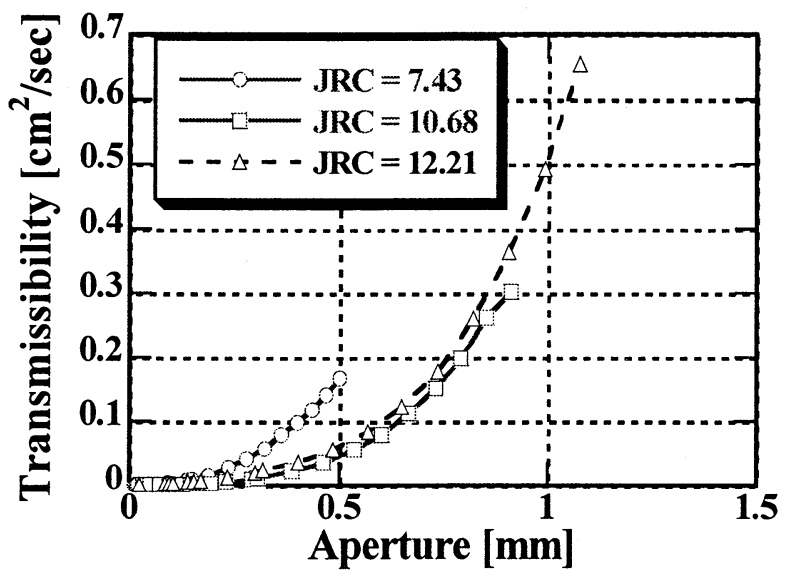

Fig.3 The variation of joint transmissibility with respect to aperture size

\section{Numerical modeling using the depth-averaged model}

\subsection{Problem formulation}

It is assumed that flow occurs between two reservoirs maintaining constant head difference $(\Delta H)$ refer Fig.4 (a) this is based on the experimental setup Fig.1. The fracture aperture is expressed as the difference between the ceiling and the bottom bed elevations, $Z_{s}$, and $Z_{b}$ (Fig. 4(b)).

\subsection{Governing Equations}

The governing equations consists of continuity and momentum equations of plane 2-D flows, obtained by integrating the 3-D continuity and Reynolds equations between the idealized bottom bed and the idealized ceiling (figure $4 \mathrm{~b}$ ) based on Cartesian coordinate system. 

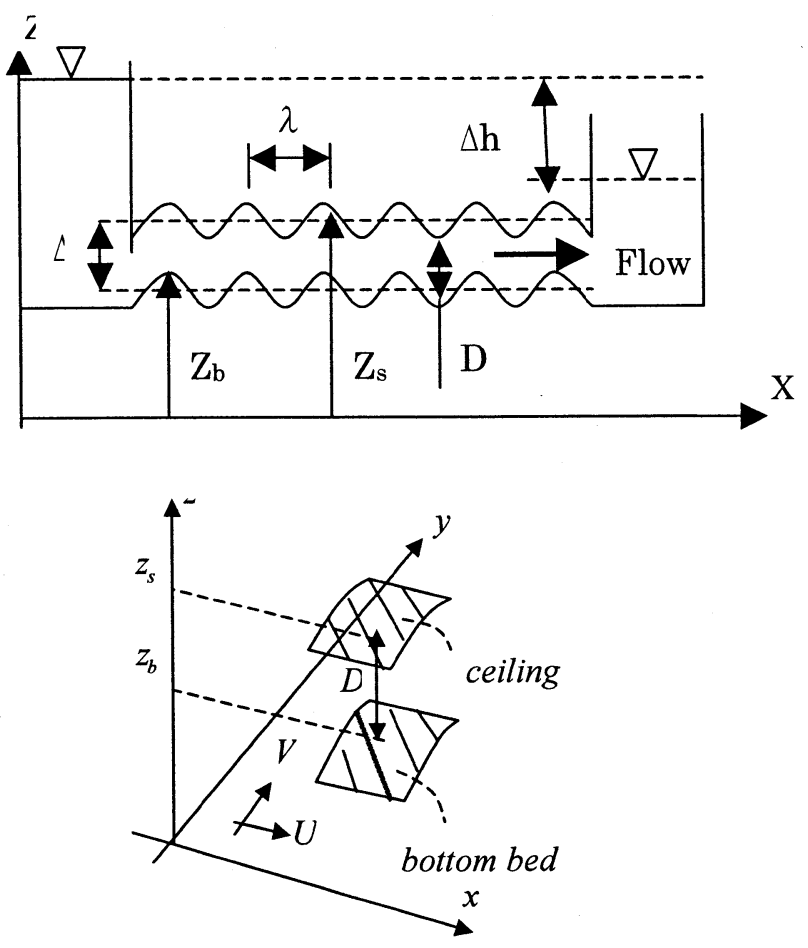

(b) Set up of coordinate system

Fig. 4 Idealized flow and coordinate setup

Continuity Equation

$\frac{\partial D U}{\partial x}+\frac{\partial D V}{\partial y}=0$

Momentum Equations

$\mathrm{x}$-direction

$\frac{\partial U D}{\partial t}+\beta \frac{\partial D U^{2}}{\partial x}+\beta \frac{\partial D U V}{\partial y}=-D \frac{\partial}{\partial x}\left(\frac{P_{D}}{\rho}\right)-g D \frac{\partial\left(Z_{b}+D\right)}{\partial x}$

$-\frac{\tau_{b x}}{\rho} \sqrt{1+\left(\frac{\partial Z_{b}}{\partial x}\right)^{2}+\left(\frac{\partial Z_{b}}{\partial y}\right)^{2}}-\frac{\tau_{s x}}{\rho} \sqrt{1+\left(\frac{\partial Z s}{d x}\right)^{2}+\left(\frac{\partial Z_{S}}{\partial y}\right)^{2}}$

$+\frac{\partial}{\partial x}\left(v D \frac{\partial U}{\partial x}\right)+\frac{\partial}{\partial y}\left(v D \frac{\partial U}{\partial y}\right)$

$\mathrm{y}$-direction

$\frac{\partial V D}{\partial t}+\beta \frac{\partial D U V}{\partial y}+\beta \frac{\partial D U V^{2}}{\partial x}=-D \frac{\partial}{\partial y}\left(\frac{P_{D}}{\rho}\right)-g D \frac{\partial\left(Z_{b}+D\right)}{\partial y}$

$-\frac{\tau_{b y}}{\rho} \sqrt{1+\left(\frac{\partial Z_{b}}{\partial x}\right)^{2}+\left(\frac{\partial Z_{b}}{\partial y}\right)^{2}}-\frac{\tau_{s y}}{\rho} \sqrt{1+\left(\frac{\partial Z_{s}}{d x}\right)^{2}+\left(\frac{\partial Z_{S}}{\partial y}\right)^{2}}$

$+\frac{\partial}{\partial x}\left(v D \frac{\partial V}{\partial x}\right)+\frac{\partial}{\partial y}\left(v D \frac{\partial V}{\partial y}\right)$

where; $\tau_{b x}, \tau_{b y}, \tau_{s x}$ and $\tau_{s y}:$ shear vector on the wall;

$(U, V)$ : depth averaged velocities; $P_{D}$ : pressure at the top boundary; $\beta$ : momentum correction factor; $v$ : coefficient of kinematics viscosity; depth (D) $=Z_{s}-Z_{b}$; g: gravitational acceleration.

The wall shear stresses $\left(\tau_{b x}, \tau_{b y}, \tau_{s x}\right.$, and $\left.\tau_{s y}\right)$ are calculated from resistance law of laminar flow.

$\frac{\tau_{b x}}{\rho}=\frac{\tau_{s x}}{\rho}=6 \frac{\nu U}{D} ; \quad \frac{\tau_{b y}}{\rho}=\frac{\tau_{s y}}{\rho}=6 \frac{\nu V}{D}$.

\subsection{Numerical Procedure}

The standard numerical method for incompressible fluids (HSMAC) is used ${ }^{8}$. It is assumed that at the initial conditions the discharge flux vector $M(=U D), N(=V D)$ and pressure $P_{D}$ at time $t=n \Delta t$ are known. The hydraulic variables $M^{*}$ and $N^{*}$ at time step $t=(n+1) \Delta t$ are then calculated as follows:

(i) First the hydraulic variables $N^{*}$ and $M^{*}$ are calculated using the following relations

$\frac{M^{*}-M^{n}}{\Delta t}=-(\text { inertia })^{n}-(\text { pressure })^{n}-(\text { wallshear })^{n}$
$\frac{N^{*}-N^{n}}{\Delta t}=-(\text { inertia })^{n}-(\text { pressure })^{n}-(\text { wallshear })^{n}$

(ii) Then the pressure $P_{D}$ is corrected by using eq. (5.)

$P_{D i, j}^{*}=P_{D i, j}^{n}+\delta P_{D}^{*}$

Where $\delta P_{D}^{*}$ for $P_{D i, j}$ is calculated using eq. (6)

$\delta^{*} P_{D i, j}=-\frac{\omega \varepsilon_{i, j}^{*}}{2 g D_{(i, j)} \Delta t\left(\frac{1}{\Delta x^{2}}+\frac{1}{\Delta y^{2}}\right)}, \quad \omega=0.5$

$\varepsilon^{*}=\frac{M_{i+1, j}^{*}-M_{i, j}^{*}}{\Delta x}+\frac{N_{i, j+1}^{*}-N_{i, j}}{\Delta y}$

(iii) $M^{*}$ and $N^{*}$ are then re-calculated by substituting the value of $P_{D}{ }^{*}$ to eq.(4). This process is repeated until the error $\left|\varepsilon^{*}\right|$ satisfy the criteria. Then $M^{*}$ and $N^{*}$ are considered to be variables at time step $t=$ $(n+1) \Delta t,\left(M^{n+1}, N^{n+1}\right)$.

\section{Model Verification}

The numerical model is verified by comparing the calculated results to analytical solution under the simple hydraulic conditions.

In this case an idealized sinusoidal surface joint roughness of rock is assumed. Basing on the fact that the flow considered in this study occurs under small amplitude-to-wavelength ratio, it is expected the flow behavior is close to unidirectional laminar flow. This allows verifying the model using one-dimensional analytical solution.

Note that different patterns of an idealized rock fracture are obtained by changing the phase angle of ceiling boundary eq. 9 . 


\subsection{Analytical Solution}

The analytical solution is based on solving the 1-D continuity and momentum equations reduced from 2-D equations (1) and (2). The following assumption is made in the solution herein obtained: It is assumed that the flow is under fully developed steady state condition.

$1-\mathrm{D}$ Continuity equation

$\frac{\partial U D}{\partial x}=0$

1-D Momentum equation

$-\beta \frac{\partial D U^{2}}{\partial x}=-D \frac{\partial}{\partial x}\left(\frac{P_{D}}{\rho}\right)-g D \frac{\partial\left(D+Z_{b}\right)}{\partial x}$

$-\frac{\tau_{b x}}{\rho} \sqrt{1+\left(\frac{\partial Z_{b}}{\partial x}\right)^{2}}-\frac{\tau_{s x}}{\rho} \sqrt{1+\left(\frac{\partial Z s}{d x}\right)^{2}}$

$+\frac{\partial}{\partial x}\left(v D \frac{\partial U}{\partial x}\right)$

In this case the bottom bed and the ceiling boundaries $\left(Z_{b}, Z_{s}\right)$ are expressed by the following relations:

$Z_{s}=A_{S} \cos \left(n x+\phi_{s}\right)+\Delta ; Z_{b}=A_{b} \cos (n x) ;$

Where; $A_{s}$ and $A_{b}$ : wave amplitudes of the bottom bed and ceiling respectively; $\phi_{\mathrm{s}}$ : profile's roughness phase angle; $\mathrm{n}$ : wave number.

It is also assumed that the pressure and shear stress variations consist of two components expressed by equation (10).

$P_{D}=P_{o}+P^{\prime} ; \quad \tau_{b x}=\tau_{s x}=\tau_{o}+\tau^{\prime}$

Where $P_{o}, \tau_{o}=$ pressure and skin shear component for undisturbed flow (parallel plate flow); $P^{\prime}, \tau^{\prime}=$ wave induced pressure and wall shear respectively.

In the previous studies of flows past wavy walls ${ }^{9,10}$, it was found that there exists a linear response for both pressure and shear variation for wavy profiles with amplitude-to-wavelength ratio $(2 a / \lambda) \leq 0.0125$.

Basing on this fact the spatial variation of pressure $P^{\prime}$ is assumed to be expressed by eq. (11), while the spatial variation of skin shear is added in the shear stress term in eq. 8 by incorporating the effect of joint surface roughness variation $\frac{\partial Z_{s}}{\partial x}$ and $\frac{\partial Z_{b}}{\partial x}$.

$\frac{P^{\prime}}{\rho g}=\left|A_{p}\right| \cos \left(n x+\phi_{p}\right)+C$

where; $A_{p}$ : Pressure amplitude; $n$ : wave number $(2 \pi / \lambda)$; $\dot{\phi}_{p}$ : pressure phase angle; $C$ : constant.

After substituting the expression for $P_{D}$ to eq. (8), it follows that:-

$$
\begin{aligned}
& \beta \frac{q^{2}}{D^{3}} \frac{\partial D}{\partial x}+g S-\frac{\partial}{\partial x}\left(\frac{P^{\prime}}{\rho}\right)-g \frac{\partial\left(D+Z_{b}\right)}{\partial x} \\
& -6 \frac{v q}{D^{3}}\left(\sqrt{1+\left(\frac{\partial Z_{b}}{\partial x}\right)^{2}}+\sqrt{1+\left(\frac{\partial Z_{s}}{d x}\right)^{2}}\right) \\
& +\frac{v q}{D^{3}} \frac{\partial}{\partial x}\left(\frac{\partial D}{\partial x}\right)^{2}-\frac{v q}{D^{2}} \frac{\partial^{2} D}{\partial x}=0
\end{aligned}
$$

Where; $-\frac{\partial}{\partial x}\left(\frac{P_{o}}{\rho}\right)=S g ; \quad q=U D ; \quad S$ : average pressure gradient.

Further, on substitution of expressions for $Z_{s}, Z_{b}, D$ and $P^{\prime}$ to eq. (12) and rearrange the like terms for $\cos (n x), \sin (n x), \cos (2 n x), \sin (2 n x), \cos (3 n x), \sin (3 n x)$, $\cos (4 n x)$ and $\sin (4 n x)$ eq. (12) is then written in the following general form;

$$
\begin{aligned}
& f_{1} \cos (n x)+f_{2} \sin (n x)+f_{3} \cos (2 n x)+f_{4} \sin (2 n x)+ \\
& f_{5} \cos (3 n x)+f_{6} \sin (3 n x)+f_{7} \cos (4 n x)+ \\
& f_{8} \sin (4 n x)+f_{0}=0
\end{aligned}
$$

Where: $f_{k}=f\left(q, A_{p}, \phi_{p}, \phi_{s}, n, A_{s}, A_{b}\right.$, and $\left.S\right)$

Where; $k=0 \ldots 8$

Keeping the mean pressure gradient $S$ constant, variables $q, A_{p}$ and $\phi_{p}$ are calculated from simultaneous equations obtained by equating the coefficients for sine, cosine and constant term $\left(f_{n^{\prime} s}\right)$ of equation (13) to zero. In this case a set of equations (14) have been solved.

$f_{0}: a_{1} q+a_{2} A_{p} \cos \left(\phi_{p}\right)+a_{3} A_{p} \sin \left(\phi_{p}\right)+a_{4}=0$

$f_{1}: b_{1} q^{2}+b_{2} q+b_{3} A_{p} \cos \left(\phi_{p}\right)+b_{4} A_{p} \sin \left(\phi_{p}\right)+b_{5}=0$ (14)

$f_{2}: c_{1} q^{2}+c_{2} q+c_{3} A_{p} \cos \left(\phi_{p}\right)+c_{4} A_{p} \sin \left(\phi_{p}\right)+c_{5}=0$

The coefficients in eq.(14) are given in appendix.

The constant ' $C$ ' is obtained by applying the boundary condition; $x=0, P^{\prime}=0$ to eq. (11). In this calculation the following flow parameters are used: - amplitude -towavelength ratio $(2 a / \lambda)=0.001$ to 0.004 (where $\mathrm{a}=$ amplitude of the bottom bed and ceiling); phase angle $\left(\phi_{\mathrm{s}}\right)$ is varied between 0.0 and $2 \pi$; the mean pressure gradient $(S=\Delta H / L)=0.1 \mathrm{~m}$ and the averaged rocky joint aperture $(\Delta)=2 m m$ refer Fig. 4 a.

\subsection{Comparison between analytical and numerical results}

The results of numerical and analytical results presented herein, are based on an idealized sinusoidal rock joint roughness. 


\section{(1) Discharge (hydraulic conductivity) variation}

The variation of discharge due to different phase angles $\left(\phi_{\mathrm{s}}\right)$ while keeping the amplitude -to-wavelength ratio $(2 a / \lambda)$ at 0.001 and 0.002 is presented in Fig. 5. It is observed that for rock joint roughness with phase angle $\left(\phi_{\mathrm{s}}\right)=0.0$, the dimensionless discharge $\mathrm{q} / \mathrm{q}_{\mathrm{o}} \quad\left(\mathrm{q}_{\mathrm{o}}-\right.$ discharge for an equivalent parallel plate flow) $\approx 1.0$. This implies, at this rock joint geometry the discharge is almost equal to that of flow between parallel plate with aperture $(\Delta)=2 \mathrm{~mm}$. Thereafter the discharge decreases with increase of phase angle $\left(\phi_{\mathrm{s}}\right)$ and become minimum at for joint roughness with $\phi_{\mathrm{s}}=\pi$ from both numerical and analytical solution with discharge reduction of about $11.6 \%$.

It is important to note that there exists good agreement between numerical and analytical solution. However, the discharge profile from analytical solution is symmetry about the axis through $\phi_{\mathrm{s}}=\pi$, while that from numerical solution is slightly skewed to the right.

Of a particular interest is the observation that increase of amplitude -to-wavelength ratio $(2 a / \lambda)$ from 0.001 to 0.002 caused a proportionate decrease of discharge while maintaining the variation trend. This is seen clearly in Fig.6 (numerical results), where the increase of amplitude -to-wavelength ratio $(2 a / \lambda)$ from 0.001 to 0.004 for phase angles $\left(\phi_{\mathrm{s}}\right) 0.0,0.6 \pi, \pi$ and $1.4 \pi$ resulted into a proportionate decrease of discharge. This points out that there exists an increase of flow resistance with increase amplitude-to- wavelength ratio of the rock joint roughness (increase of roughness). Also it is important to note that for the joint roughness with phase angle $\left(\phi_{\mathrm{s}}\right)=\pi$ the reduction of discharge at amplitude-to-wavelength ratio $=0.004$ is about $40 \%$.

This implies that, high resistance to flow is observed for the rock joint roughness with phase angle $\left(\phi_{\mathrm{s}}\right)=$ $\pi$.

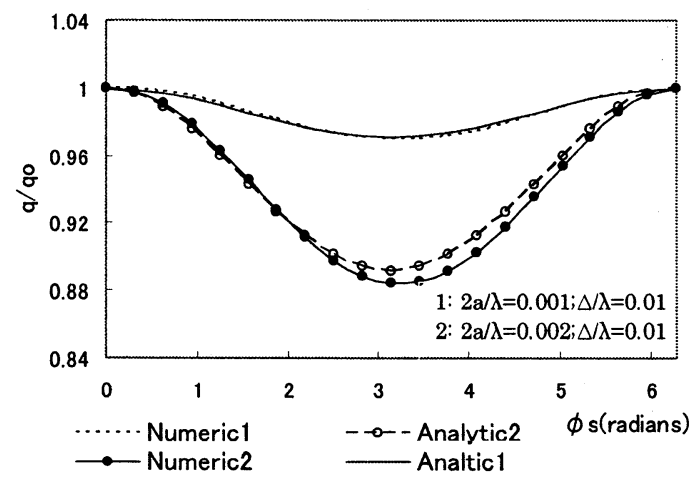

Fig. 5 The variation of discharge with different profile's geometry

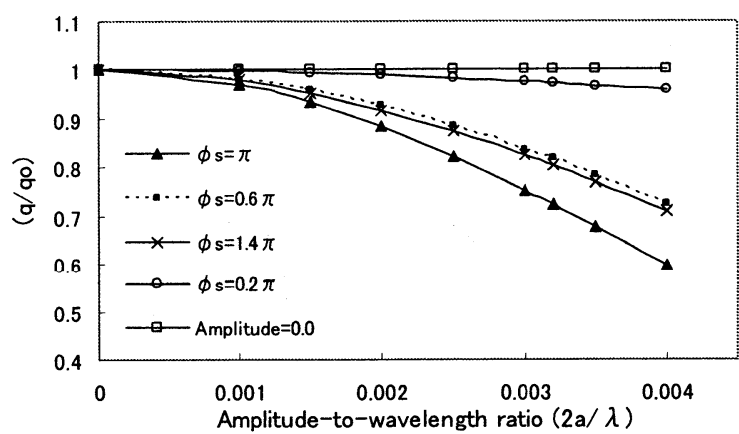

Fig. 6 Variation of discharge with amplitude wavelength ratio

\section{(2) Pressure distribution}

Using boundary conditions in section 4.1 , the constant ' $C$ ' of the periodic pressure term $\left(P^{\prime}\right)$ is expressed by the relation $C=-A_{p} \cos \left(\phi_{p}\right)$ for all range of phase angles. Eq. (10) becomes:

$$
\frac{P^{\prime}}{\rho g}=A_{p}\left\{\cos \left(n x+\phi_{p}\right)-\cos \left(\phi_{p}\right)\right\}
$$

It is observed that the pressure variation along the sinusoidal fracture is periodic with wavelength equal to that of the rock joint roughness from both numerical and analytical results as shown in Fig.7a. This is analogy to the results presented from different studies on pressure variation of flows in wavy bed profiles ${ }^{9,10}$. When the phase angle $\left(\phi_{\mathrm{s}}\right)=0.0$ the existence of periodic variation disappears, that is, the pressure distribution resembles that of flow between parallel plates. This is in agreement to the results observed for discharge variation.

Investigating the periodic component of pressure variation for one wavelength between $\mathrm{L}=0.35$ to $\mathrm{L}=$ 0.55 (Fig. 7b, c, d) it is evident that the pressure amplitude increase when phase angle $\left(\phi_{s}\right)$ is increased and attains maximum value for the fracture with phase angle $\left(\phi_{\mathrm{s}}\right)=\pi$.

\section{(3) Shear stress distribution}

It is observed that there exists good agreement between the analytical and numerical results (Fig.8a, b, and c). For the rock joint roughness with phase angle $\phi_{\mathrm{s}}=\pi$ the maximum of shear stress variation remains near the peaks while the minimum is near the troughs Fig.8 (a).

There is no variation of shear stress for the rock joint roughness with phase angle $\left(\phi_{\mathrm{s}}\right)=0.0$ This is identical to the shear distribution along parallel plate flow. 


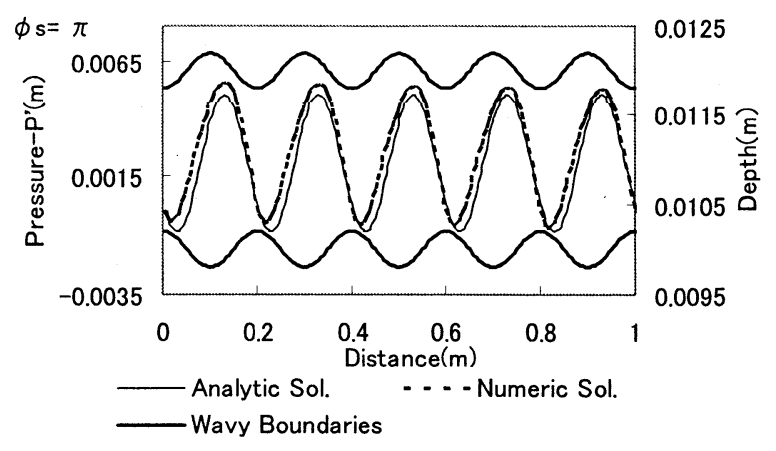

(a)

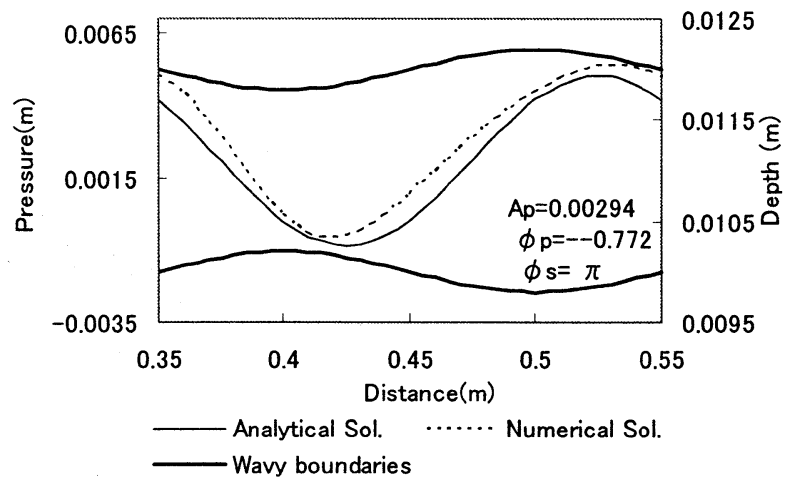

(b)

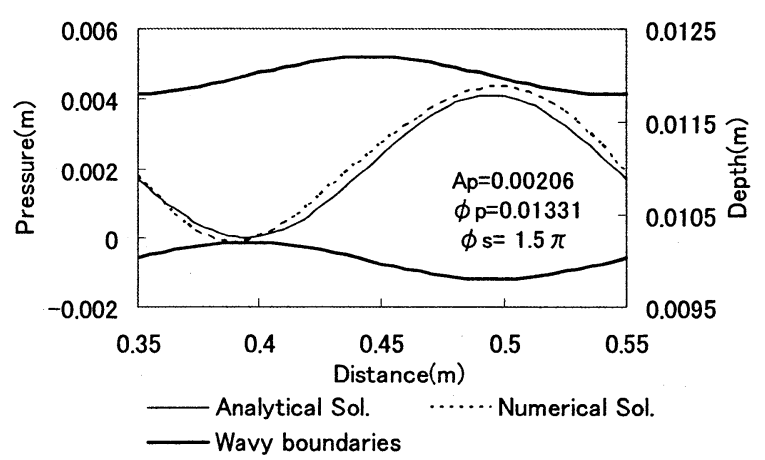

(c)

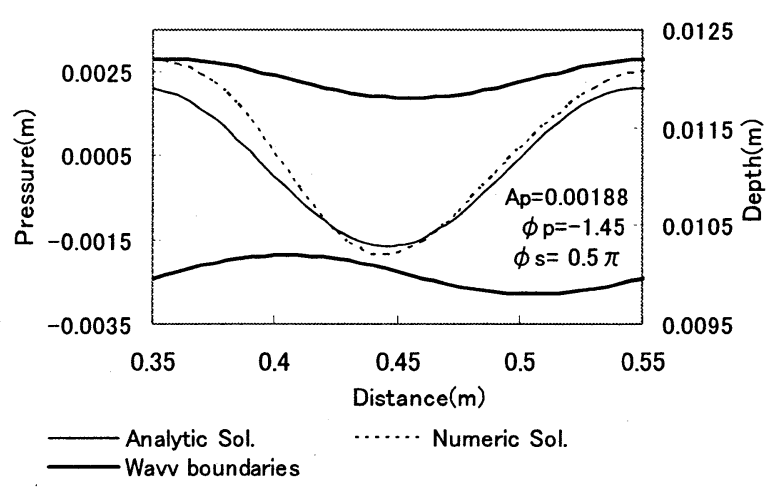

(d)

Fig. 7 Periodic component of pressure variation

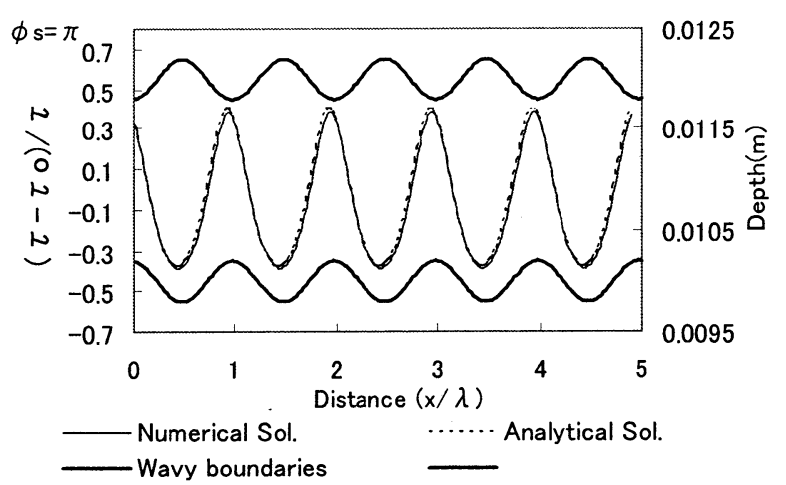

(a)

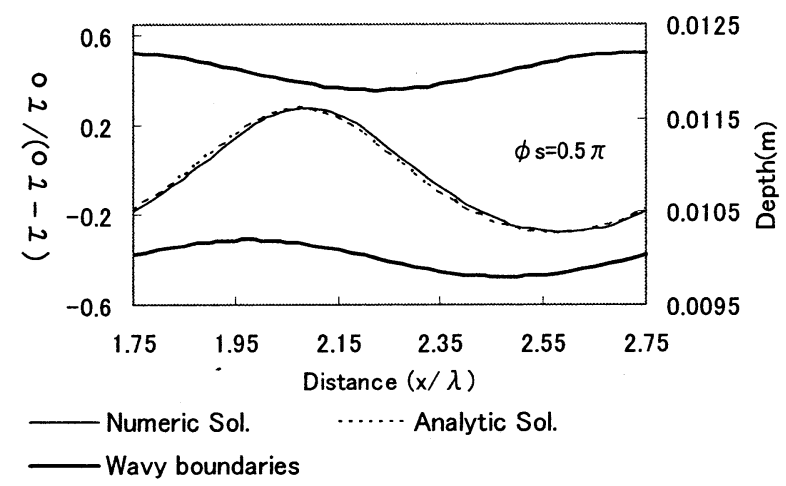

(b)

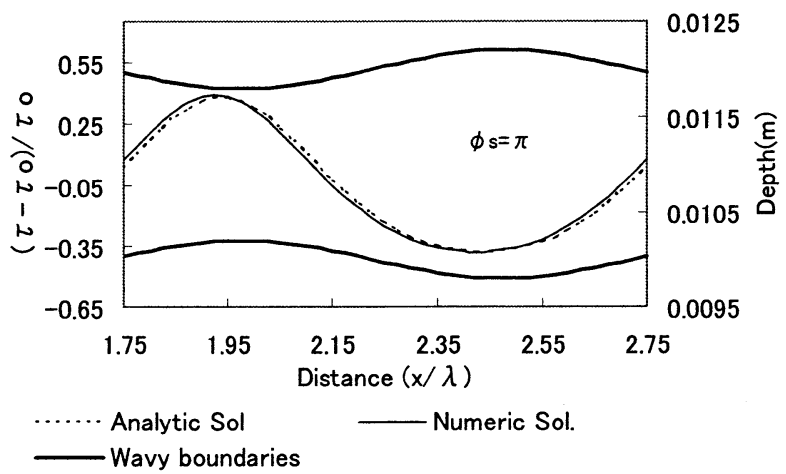

(c)

Fig. 8 Variation of shear stress along the rocky joint ( $\tau_{0}$ - skin shear for an equivalent parallel plate)

\section{Application of numerical model to experimental rock joints}

The results presented herein based on the input parameters from experimental specimen, that is $Z_{s}, Z_{b}$ and $\Delta H=1.0 \mathrm{~m}$.

Fig. 9 shows the surface roughness distribution of the specimens herein considered. Fig. 9 shows the surface roughness distribution of the specimens herein considered. The aperture size ranged between $0-1.5 \mathrm{~mm}$ for all specimens. However it can be noted that the roughness distribution varied differently from on specimen to the other. Note that the sections A-A and B-B are taken mid-way along the width of the specimens. 

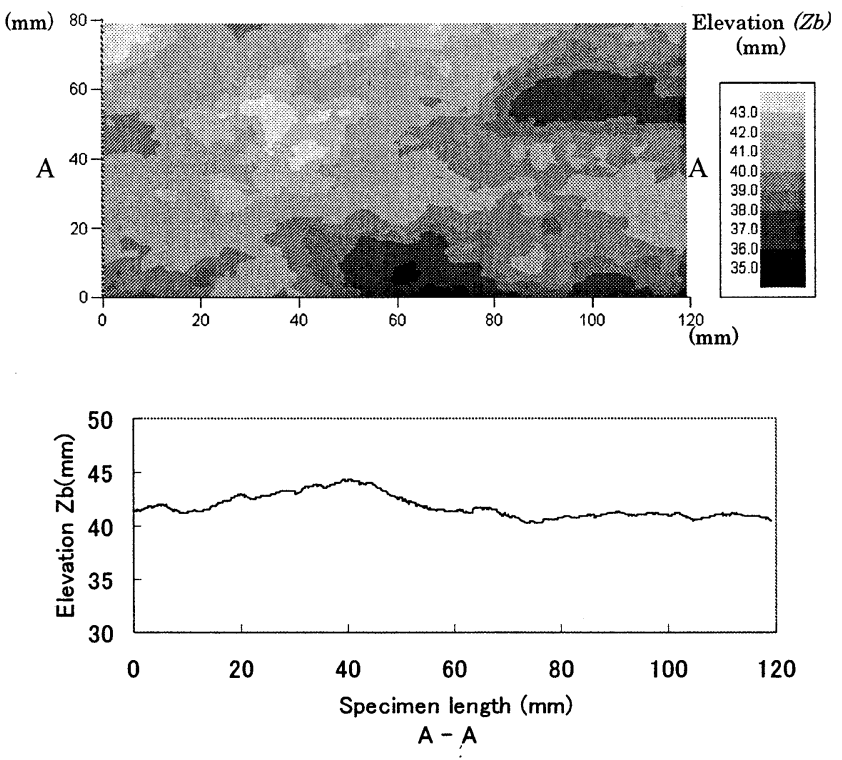

(a) Specimen A-L02
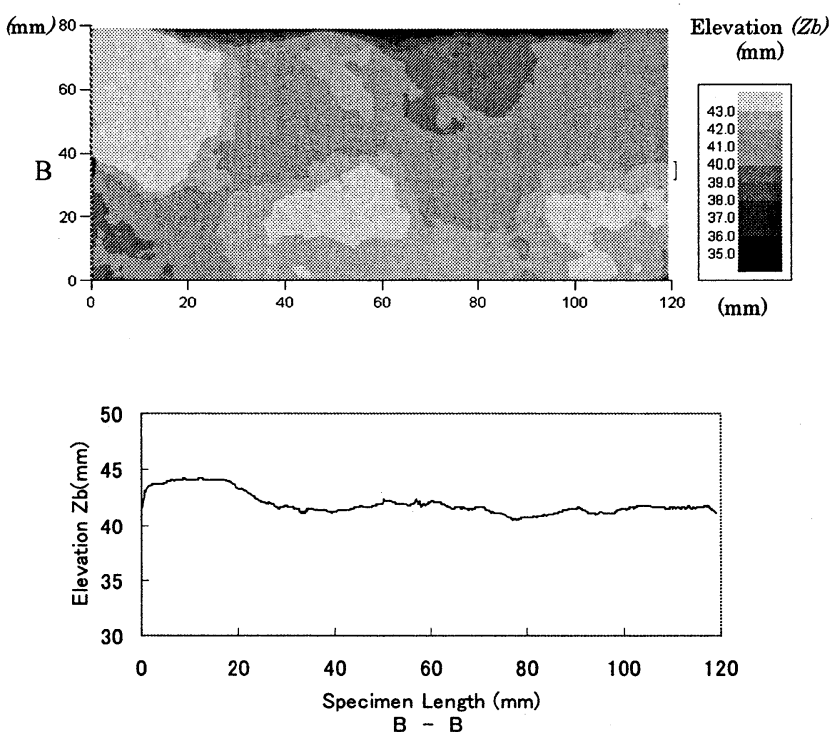

(b) Specimen C-L02

Fig. 9 Surface roughness distribution

The comparisons of the experimental results and the simulation results for shear displacement -transmissibility relation are shown in Fig.10. It is observed that results of two-dimensional flow model exhibits good agreement with the experimental results for small shear displacements. However, there are noticeable disagreement at large shear displacements for specimens A-L02 and C-L02, where the results of 2-D model become smaller than that of the experiment.

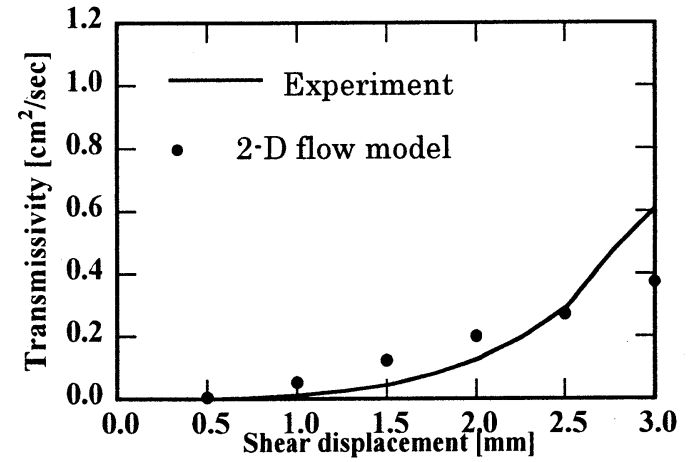

(a) Specimen A-L02

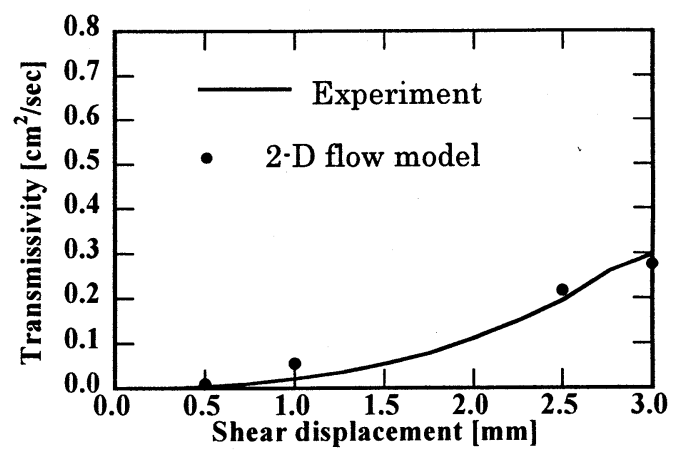

(b) Specimen B-L02

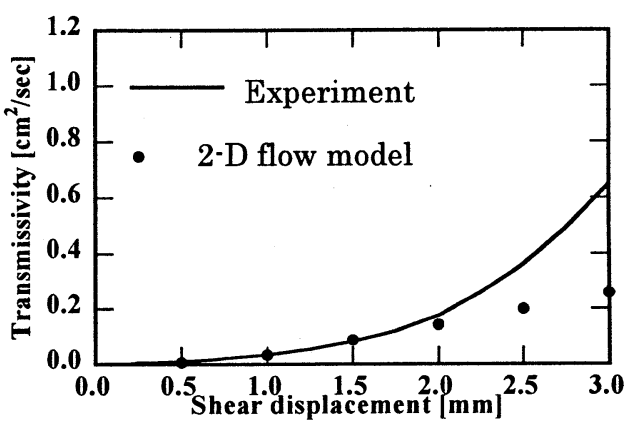

(c) Specimen C-L02

Fig.10 Comparison of the experimental and numerical results

From Fig.11 it is observed that for the specimens A-L02 and C-L02 the estimated dilation from the numerical model is smaller than that of experiments. Dilation is the vertical movement of one part of the specimen tending to move away from the other due to shear displacement as result of misalignment of the rock joint. Therefore for specimens A-L02 and C-L02, it is expected that the estimated aperture by numerical simulation to be smaller that the actual value. This can results into underestimating transmissibility at higher values of shear displacement. Also during experiment there is a possibility of having joint roughness 


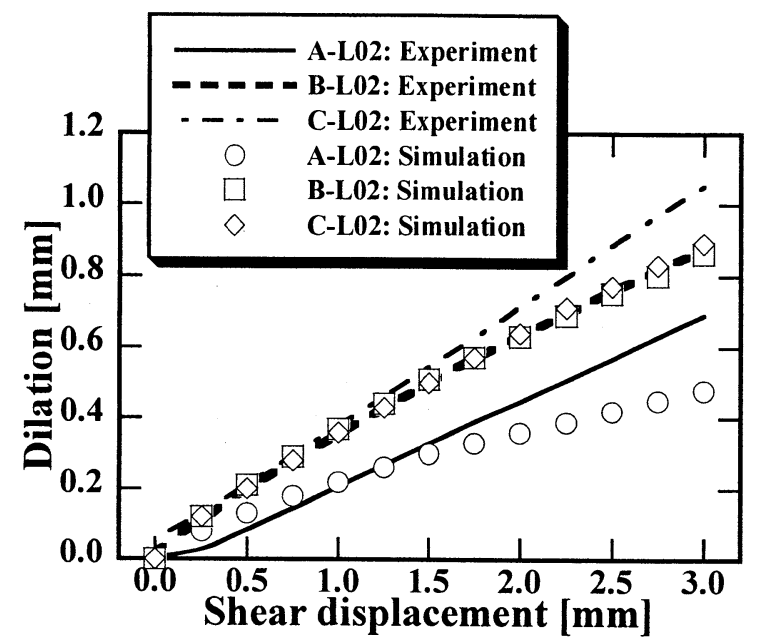

Fig.11 The comparison of the experiments and the simulations on shear displacement-dilation relation

deformation at larger shear displacements, which could not be accommodated in the numerical model.

Fig. 12 shows the flow vector distribution simulated by the model at $2.5 \mathrm{~mm}$ shear displacement. It is observed that the model has been able to simulate the channeling effect across the rock joint specimens.

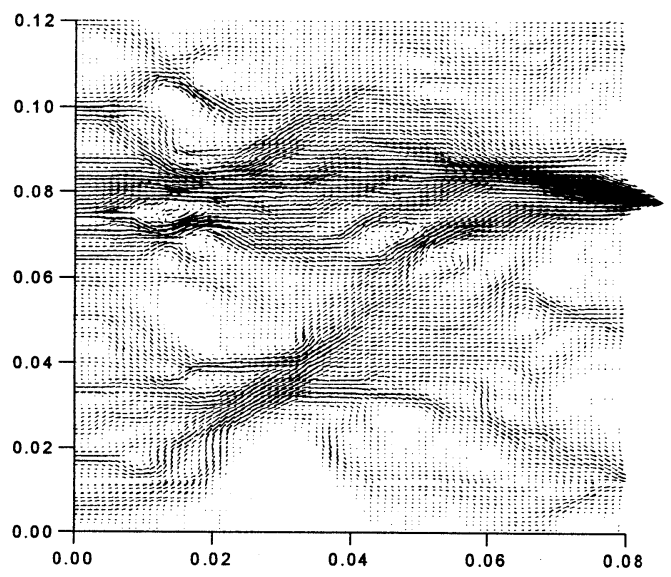

(a) Specimen A-L02

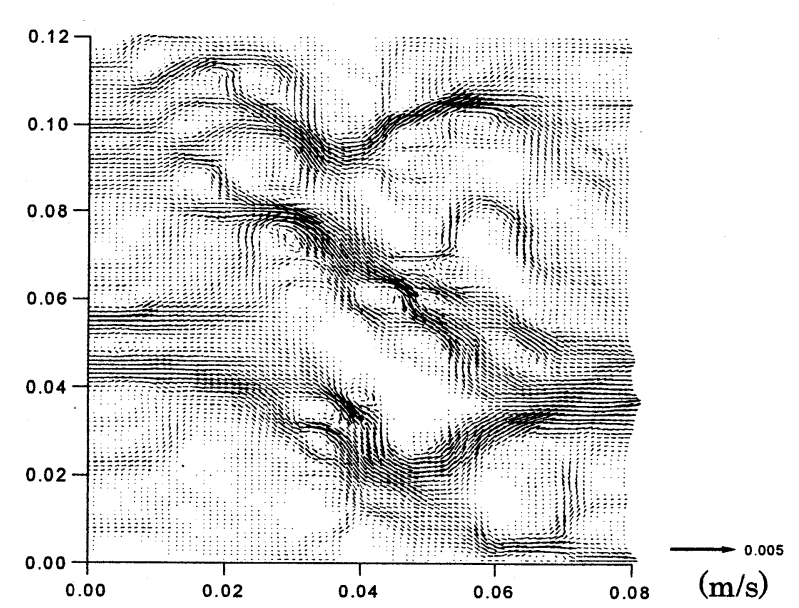

(b) Specimen C-L02

Fig.12 Distribution of discharge vector on rock joints

\section{CONCLUSION}

Basing on the results from an idealized sinusoidal rock roughness, it is evident that the resistance to flow in rock joint is largely influence by both longitudinal distribution of the roughness (surface roughness) and the size of aperture.

The depth -averaged flow model proposed herein has shown its ability in simulating the flows in rock joint especially for small shear displacement. However, more research is needed to find out on how the effect deformation of rock joint roughness during shear displacement can be accommodated in the model. It is also needed to develop the refined depth averaged flow model considering the effect of circulation behind spatial boundary variations to predict the flow and the resistance precisely.

\section{Appendix}

Symbols used in analytical solution of one-dimensional flow model

$$
\begin{aligned}
a_{1}= & -6 v\left(2+\frac{1}{4} \omega_{b}^{2}+\frac{1}{4} \omega_{s}^{2}-\frac{3}{64}\left(\omega_{b}^{4}+\omega_{s}{ }^{4}\right)\right) \\
& +v\left(\omega_{s}^{2}+\omega_{b}^{2}-2 \omega_{s} \omega_{b} \cos \left(\phi_{s}\right)\right) \\
a_{2}= & \frac{g n}{2}\left(\alpha_{6} \sin \left(2 \phi_{s}\right)-\alpha_{1} \sin \left(\phi_{s}\right)+\frac{\alpha_{8}}{2} \sin \left(\phi_{s}\right)\right) ; \\
a_{3}= & \frac{g n}{2}\left(\alpha_{1} \cos \left(\phi_{s}\right)-\alpha_{3}-\alpha_{6} \cos \left(2 \phi_{s}\right)+\alpha_{8} \cos \left(\phi_{s}\right)\right) \\
a_{4}= & g\left(S \alpha_{10}+\frac{\omega_{s}}{2}\left(\left(\alpha_{6}-\alpha_{3}\right) \sin \left(\phi_{s}\right)+\alpha_{8} \sin \left(2 \phi_{s}\right)\right) ;\right. \\
b_{1}= & -1.2 \omega_{s} \sin \left(\phi_{s}\right) ; b_{2}=v n\left(\omega_{s} \Delta \cos \left(\phi_{s}\right)-\omega_{b} \Delta\right) \\
b_{3}= & \frac{g n}{2}\left(-\alpha_{5} \sin \left(2 \phi_{s}\right)+\alpha_{7} \sin \left(\phi_{s}\right)\right) \\
b_{4}= & \frac{g n}{2}\left(\alpha_{5} \cos \left(2 \phi_{s}\right)-\alpha_{7} \cos \left(\phi_{s}\right)+\alpha_{9}+2 \alpha_{10}\right) \\
b_{5}= & S g\left(\alpha_{1} \cos \left(\phi_{s}\right)-\alpha_{3}-\alpha_{6} \cos \left(2 \phi_{s}\right)+\alpha_{8} \cos \left(\phi_{s}\right)\right) \\
& \left.+\frac{A_{s} g n}{2}\left(2 \alpha_{10}-\alpha_{5}\right) \sin \left(\phi_{s}\right)+\alpha_{9} \sin \left(\phi_{s}\right)\right) \\
c_{1}= & 1.2\left(\omega_{b}-\omega_{s} \cos \left(\phi_{s}\right) \quad ; \quad c_{2}=-v \omega_{s} n \Delta \sin \left(\phi_{s}\right) ;\right. \\
c_{3}= & \frac{g n}{2}\left(-\alpha_{5} \cos \left(2 \phi_{s}\right)+\alpha_{7} \cos \left(\phi_{s}\right)-\alpha_{9}+2 \alpha_{10}\right) \\
c_{4}= & \frac{g n}{2}\left(\alpha_{7} \sin \left(\phi_{s}\right)-\alpha_{5} \sin \left(2 \phi_{s}\right)\right) ;
\end{aligned}
$$




$$
\begin{aligned}
c_{5}= & S g\left(\alpha_{6} \sin \left(2 \phi_{s}\right)-\alpha_{1} \sin \left(\phi_{s}\right)+\alpha_{8} \sin \left(\phi_{s}\right)\right) \\
& \left.+\frac{A_{s} g n}{2}\left(2 \alpha_{10}-\alpha_{5}\right) \cos \left(\phi_{s}\right)+\alpha_{7}-\alpha_{9} \cos \left(\phi_{s}\right)\right)
\end{aligned}
$$

where;

$$
\begin{aligned}
& \omega_{b}=\frac{2 A_{b} \pi}{\lambda} ; \quad \omega_{s}=\frac{2 A_{s} \pi}{\lambda} ; \quad n=\frac{2 \pi}{\lambda} \\
& \alpha_{1}=\frac{3}{4} A_{s}{ }^{3}+3 A_{s} \Delta^{2}+\frac{3}{2} A_{s} A_{b}{ }^{2} ; \quad \alpha_{2}=\frac{1}{4} A_{s}{ }^{3} ; \\
& \alpha_{3}=\frac{3}{4} A_{b}{ }^{3}+3 A_{b} \Delta^{2}+\frac{3}{2} A_{s}{ }^{2} A_{b} ; \quad \alpha_{4}=\frac{1}{4} A_{b}{ }^{3} ; \\
& \alpha_{5}=\frac{3}{2} A_{s}{ }^{2} \Delta ; \quad \alpha_{6}=\frac{3}{4} A_{s}{ }^{2} A_{b} ; \quad \alpha_{7}=3 A_{b} \Delta A_{s} \\
& \alpha_{8}=\frac{3}{4} A_{s} A_{b}{ }^{2} ; \quad \alpha_{9}=\frac{3}{2} A_{b}{ }^{2} \Delta ; \\
& \alpha_{10}=\frac{3}{2} A_{s}{ }^{2} \Delta-3 A_{b} \Delta A_{s} \cos \left(\phi_{s}\right)+\frac{3}{2} A_{b}{ }^{2} \Delta+\Delta^{3} \\
& g=9.81 \mathrm{~m} / s^{2}
\end{aligned}
$$

\section{REFERENCES}

1) Elsworth, D. and Goodman, R.E., Characterization of Rock Fissure Hydraulic Conductivity Using Idealized Wall Roughness Profiles, J. Rock Mech. Min. Sc. \& Geomech. Vol. 23, No. 3, pp 233-243, 1986.

2) Tsang Y.W and Witherspoon P.A. The dependence of fracture mechanical and fluid properties on fracture roughness and sample size. J. Geophys. Res. Vol. 88(B3), 2359-2366, 1983.

3) Kishida, K., T. Adachi and K. Tsuno. 2001. Modeling of the shear behavior of rock joints under constant normal confining conditions. Rock Mechanics in the National Interest, Proceedings of the 38th U.S. Rock Mechanics Symposium, Elsworth, Tinucci \& Heasly, (eds). 791 - 798. Rotterdam: Balkema.
4) Kishida, K., H. Taniya, S. Nakashima and T. Adachi. 2002. Shear behavior of rock joints under constant normal stiffness conditions. NARMS-TAC 2002, Mining and Tunneling Innovation and Opportunity, Hammah et al. (eds). 53-61. University of Toronto.

5) Ohnishi, Y., T. Yano, H. Iwamoto and N. Nishikawa. 2000. The history of the research and development of the apparatuses measuring both the shear strength and the roughness profile of rock joints. Journal of Geotechnical Engineering, JSCE. No.645/III-50. 307-320 (in Japanese).

6) Takagi, K., Y. Ohnishi, H. Ohtsu, S. Nishiyama and T. Yano. 2002. A study of hydraulic characteristics of rock joints under shear deformation. Proc. of the $57^{\text {th }}$ annual meeting, JSCE. III-459 (in Japanese).

7) Barton N.R, Choubey V., The shear strength of rock joints in theory and practice. Rock Mech. 10: 1-65 (1977)

8) Hosoda, T., Inoue, K. and Tada, A. Hydraulic transient with propagation of interface between open-channel free surface flow and pressurized flow, Proc. International Symp. on Computational Fluid Dynamics, Vol. I, Sendai, 291-296, 1993.

9) Patel, V.C., Chon, J.T. and Yoon, J.Y., Laminar flow over wavy walls, J. Fluid Eng. Vol. 113, pp 574-578, 1991.

10) Patel, V.C., Chon, J.T. and Yoon, J.Y., Turbulent flow in a channel with a wavy wall, J. Fluid Eng. Vol. 113, pp 579-586, 1991.

11) Brown S.R., Stockman H.W. and Reeves S.J., Applicability of the Reynolds equation for modeling fluid flow between rough surfaces J. Geoph. Res. Letters Vol. 22 No. 18, pp 2537-2540, 1995.

12) Zilker, D.P, Cook, G.C. and Hanratty,T J., Influence of the amplitude of a solid wavy wall on a turbulent flow. Part 1. Non separated flows, J. Fluid Mechanics 82, pp. Vol. 29-51, 1977.

(Received: 2004, April 16) 